INPLASY

PROTOCOL

To cite: Peixoto et al. Effects of velocity loss in the squat and bench press on neuromuscular performance: a systematic review with meta-analysis. Inplasy protocol 202120083. doi:

10.37766/inplasy2021.2.0083

Received: 28 February 2021

Published: 28 February 2021

Corresponding author:

Rafael Peixoto

peixoto347@gmail.com

Author Affiliation:

Universidade de Trás-os-

Montes e Alto Douro

Support: Foundation

Science\&Technology.

Review Stage at time of this submission: Preliminary

searches.

Conflicts of interest:

None declared.

\section{Effects of velocity loss in the squat and bench press on neuromuscular performance: a systematic review with meta-analysis}

Peixoto, R'; Vilaça-Alves, J2; Afonso, J3; Canário-Lemos, R4; Rafael-Moreira, T5; Pinheiro, $\mathrm{B}^{6}$.

Review question / Objective: What are the effects of different velocity loss interventions on neuromuscular performance? Condition being studied: The chronic effect of different velocity loss protocols on neuromuscular performance on bench press and squat.

Information sources: The following databases will be consulted: Cochrane, EBSCO (Academic Search Complete, CINAHAL Plus, MEDLINE, PsycINFO, SportDISCUS), ISI Web of Knowledge, PEDro, PubMed, Scielo and Scopus. Also we will contact with authors and seek experts for any additional studies.

INPLASY registration number: This protocol was registered with the International Platform of Registered Systematic Review and Meta-Analysis Protocols (INPLASY) on 28 February 2021 and was last updated on 28 February 2021 (registration number INPLASY202120083).

\section{INTRODUCTION}

Review question / Objective: What are the effects of different velocity loss interventions on neuromuscular performance?
Rationale: The main goal of the strength and conditioning coach is to increase performance, e.g. strength and power, of their athletes controlling several training variables, such as sets, repetitions, load, among others. Velocity based training has beed a great way to control and monitor training loads as well as adjust relative 
training load. It seems there is a linear relationship between the velocity loss and the fatigue accumulated in a set of a determined exercise. Since there seems to be an increase in strength without reaching muscular failure and the time to recover seems to be lower when muscular failure is not achieved, managing velocity loss in training seems to be an optimal way of manage the total fatigue accumulated by the athletes. Several studies show that it seems to be an increase in strength and power with low values of velocity loss and it seems to be equal or sometimes higher gains, when comparing lower velocity loss with higher velocity loss. So, our goal is to understand the chronic effects of different velocity loss protocols on neuromuscular performance of the bench press and squat.

Condition being studied: The chronic effect of different velocity loss protocols on neuromuscular performance on bench press and squat.

\section{METHODS}

Search strategy: We will search with these terms ("Strength" OR "Power") AND ("Squat" OR "Bench Press") AND ("Velocity "), with no restrictions for title or abstract and no restrictions in terms of publication date. termos mesh) (limite de data).

Participant or population: Humans regardless of their training status or health condition.

Intervention: Velocity Based Training.

Comparator: Additional Velocity Based Training with a velocity loss lower to the intervention.

Study designs to be included: Randomized Controlled Trials or Randomized Trials.

Eligibility criteria: Humans regardless of their training status or health condition; Velocity Based training intervention, as long as there were more than one group and randomization was used; any effects on neuromuscular performance and have at least 4 weeks of intervention; The intervention must be made on the bench press or the squat exercise.

Information sources: The following databases will be consulted: Cochrane, EBSCO (Academic Search Complete, CINAHAL Plus, MEDline, Psycinfo, SportDISCUS), ISI Web of Knowledge, PEDro, PubMed, Scielo and Scopus. Also we will contact with authors and seek experts for any additional studies.

Main outcome(s): Progressive loading test on the Bench Press or the Squat or a Vertical Jump test.

Data management: The main author of this manuscript will conduct the first literature review and data extraction. A second author of this manuscript will independently repeat the process within the same month and the two processes will be compared to ensure that there were no mistakes in the literature search and retrieval process, as well as in the exclusion process. The remaining authors of this work will crosscheck the entire process to verify errors or inconsistencies. Then, the authors will be divided in teams of two and each team will perform, independently, the risk-of-bias analysis.

Quality assessment / Risk of bias analysis: The revised Cochrane risk-of-bias tool for randomized trials (RoB 2) will be applied to evaluate the individual studies, considering its five dimensions: bias arising from the randomization process, bias due to deviations from intended interventions, bias due to missing out- come data, bias in measurement of the outcome and bias in selection of the reported result.

Strategy of data synthesis: A systematic review will be made with the studies that respect the inclusion criteria. The risk of bias will be analysed throw the RoB 2. If a meta-analysis is possible, a minimum of three studies will be used in order to avoid small sample sizes. Pre- and Postintervention means and standard deviations (SDs) will be converted to Hedge's g effect size (ES). In case the study 
instead provides $95 \%$ confidence intervals (CIs) or standard erros of mean (SEM), means and SDs will be obtained from $95 \%$ CI or SEM. The ESs will be presented alongside $95 \% \mathrm{Cls}$ and interpreted using the following thresholds: $\mathbf{0 . 2}$, trivial; $\mathbf{0 . 2 - 0 . 6}$, small; 0.6-1.2, moderate; 1.2-2.0, large; 2.0-4.0, very large; 4.0, extremely large. Heterogeneity will be assessed using the $\mathbf{I}^{2}$ statistic, with values of $25 \%, 25-75 \%$ and $75 \%$ considered to represent low, moderate, and high levels of heterogeneity, respectively. Publication bias will be explored using the extended Egger's test.

Subgroup analysis: Using random-effects model and independent computed single factor analysis, potential sources of heterogeneity likely to influence the effects of interventions are selected a priori. As the post-intervention results of neuromuscular performance may be affected by participants training status (eg. athletes vs sedentary), health status and sex, these factors are considered as potential moderator variables. When appropriate, subgroup analyses and single training factor analyses will be divided using the median split technique, if at least three studies provide data for a given moderator. To minimize heterogeneity, median values will only be calculated considering those studies that provide data for the outcome being analysed.

Sensitivity analysis: A sensitivity analysis will be conducted using the trim and fill method. A multivariate random-effects meta-regression is planned to verify if any of the intervention variables (e.g. \% repetition maximum used, $\%$ of velocity loss used) predicts the effects of postexercise neuromuscular performance on outcome measures. Computation of metaregression will be performed with at least 10 studies per covariate. Statistical significance will be set at $p<=0.05$.

Language: No limits will be imposed.

Country(ies) involved: Portugal.

Dissemination plans: Once the systematic review and a possible meta-analyses is made, the goal will be to publish. The goal will be to finish the the systematic review and meta-analyses on the 3rd trimester of the present year.

Contributions of each author:

Author 1 - Rafael Peixoto - The author will draft the manuscript and contribute to the development of the selection criteria, and the risk of bias assessment strategy.

Email: peixoto347@gmail.com

Author 2 - Jose Vilaça-Alves - The author will read, provide feedback and approve the final manuscript.

Author 3 - Jose Afonso - The author will read, provide feedback and approve the final manuscript and contribute to the risk of bias assessment strategy.

Email: jneves@fade.up.pt

Author 4 - Rui Canário-Lemos - The author will contribute to the development of the selection criteria and will perform the search.

Email: ruimaldini27@hotmail.com

Author 5 - Bruno Pinheiro - The author provided statistical expertise.

Email:bruno.pinheiro@professor.unifametro .edu.br

Author 6 - Tiago Rafael-Moreira - The author will contribute to the development of the selection criteria and will perform the search.

Email: tromoreira@hotmail.com 